\title{
The role of imaging in head and neck cancer: An overview of different imaging modalities in primary diagnosis and staging of the disease
}

\author{
Veena Vishwanath, BSc, MBBS, FRCR!' Sarah Jafarieh, MBBS, FRCR², Agata Rembielak, BSc, MD, PhD, MA, MRCR 3.4 \\ 'Royal Preston Hospital, Preston, United Kingdom, ${ }^{2}$ Salford Royal Hospital, Salford, United Kingdom, ${ }^{3}$ The Christie NHS Foundation Trust, \\ Manchester, United Kingdom, ${ }^{4}$ The University of Manchester, Manchester, United Kingdom
}

\begin{abstract}
Radiology has an essential role in diagnosis, staging, and management in all subspecialties related to oncology. It has a broad utility from its use as an initial screening tool for cancer detection, followed by staging and surveillance of disease as well as the delivery of appropriate treatment regimens.

In order to plan a treatment, the use of different modalities of radiological imaging are a key factor for pre-treatment planning and staging of the extent of disease in accordance with tumor-node-metastasis (TNM) system for highly conformal treatments, such as brachytherapy (BT).

In this work, we present an overview of main modalities of imaging and principles of their application especially in head and neck cancer, with its diagnosis, treatment, follow-up with post-treatment changes, and overall management.

J Contemp Brachytherapy 2020; 12, 5: 512-518 DOI: https://doi.org/10.5114/jcb.2020.100386
\end{abstract}

Key words: imaging, head and neck cancer, ultrasound, CT, MRI, PET-CT.

\section{Purpose}

Head and neck cancer (HNC) is a general term with variety of subsites, such as oral cavity, sinuses, salivary glands, oropharynx, nasopharynx, hypopharynx, and larynx. These neoplasms account for $5 \%$ of cancers worldwide and are the $5^{\text {th }}$ most common cancer condition [1]. Many skin cancers also present at head and neck sites, with basal cell carcinoma as the most common form, followed by squamous cell carcinoma, and melanoma.

The use of brachytherapy (BT) has an essential role in the management of HNC due to its ability to deliver highly conformal radiation dose to the target area while minimizing treatment-related side effects, reducing morbidity, and improving function and cosmesis [2]. In HNC, BT has an established role in definitive and post-operative settings, in loco-regional recurrence, and in palliative cases. In a more advanced disease, BT treatment is often combined with external beam radiotherapy and systemic anticancer treatment (SACT), which can improve overall survival outcomes.

In order to plan a treatment, the use of different modalities of radiological imaging are a key factor for pretreatment planning and staging of the extent of disease in accordance with a tumor-node-metastasis (TNM) system for highly conformal treatment, such as BT. In this work, different modality choices are explored for each head and neck cancer subsite, along with their role in the treatment and overall management. This is an educational article intended to provide an overview of principles in head and neck imaging in general settings. The use of head and neck imaging in specific radiotherapy indication, such as imaging-based catheter verification in BT, are above the scope of this article, but are planned for subsequent follow-up papers.

\section{Imaging modalities in head and neck cancer \\ Contrast-enhanced computed tomography}

Computed tomography (CT) is widely used in oncology and can be applied to assess primary site of head and neck cancer, nodal disease, and staging as a part of the established TNM staging system.

The use of thin-slice high-resolution image acquisition allows for good quality, multiplanar reconstructed images for anatomy demonstration and treatment planning. Modern helical multidetector CT slice thickness is dependent on the type of scanner; however, collimation of 1-1.5 mm is generally used with a field of view of $16-20 \mathrm{~cm}$. Multiplanar images are reformatted at 2-3 mm thickness and in some situations, high resolution sections can be acquired as low as $0.625 \mathrm{~mm}$ based on scanner capability. 
These images are obtained under spiral technique, following injection with an intravenous contrast medium, and the use of dynamic acquisition allows up to eight images to be obtained per second $[3,4]$. Iodinated contrast and a long bolus technique can be applied, for example, $100 \mathrm{ml}$ of Omnipaque $300^{\oplus}$ at $1 \mathrm{ml} / \mathrm{s}$, with the commencement of imaging at 90-100 seconds [5]. Occasionally, CT neck angiograms in arterial phase can be performed using a bolus triggered technique. This may be of use in assessing the caliber and patency of carotid vessels, along with evaluating the nature of highly vascular tumors.

When compared to magnetic resonance imaging (MRI), CT can provide an excellent assessment of the infrahyoid neck, minimizing any artefact movement due to its rapid acquisition time. It can provide an improved assessment of bony cortex, cartilage involvement, and calcification. At the same time, it can concurrently assess the thorax for paratracheal and upper mediastinal lymph nodes, lung metastases, or synchronous primary lung lesion.

A few disadvantages of CT compared to other modalities of imaging include radiation dose burden, risk of contrast-induced nephropathy, and artefacts secondary to dental amalgam/orthopedic prostheses.

The average dose of a CT neck with IV contrast is $3.9 \mathrm{mSv}$ [6], and for CT Thorax - $8 \mathrm{mSv}$ [7]. This is compared to the average background radiation exposure of a person to $2.2 \mathrm{mSv}$ per year in the UK and $6.2 \mathrm{mSV}$ per year in the USA [8].

Omnipaque $300 \mathrm{Mg}$ Iodine $^{\circledast}$ is commonly used as the iodinated intravenous contrast medium to highlight blood vessels and to enhance soft tissue structures. In the absence of a contrast, it can be difficult to depict abnormal soft tissue and detect pathological lymph nodes, particularly throughout the neck and those within close proximity to vascular structures. However, a non-contrast scan may still be of benefit as a gross assessment of soft tissue density, detecting calcification, measurement of large neck nodal masses, in the early detection of lung nodules, and assessing background diffuse/chronic lung disease, wherever appropriate.

There are contraindications to iodinated contrast use, which include severe renal disease (estimated glomerular filtration rate - eGFR $<30 \mathrm{ml} / \mathrm{min} / 1.73 \mathrm{~m}^{2}$ ), acute liver disease, asthma, thyroid disease, dehydration, sickle cell anemia, seizures, and pheochromocytoma and allergies to contrast [9].

Barium-based contrast agents are less frequently used as a method of oral contrast for the assessment of gastrointestinal tract. However, compared to other modalities, CT has advantages in being a readily available source of imaging and provides a rapid image acquisition time, which is ideal for patients with difficulties in swallowing, breathlessness, and with a poor ability to lie flat. Example of contrast-enhanced CT neck is presented in Figure 1.

\section{Magnetic resonance imaging}

Magnetic resonance imaging (MRI) can assess pathological processes down to a molecular level due to its ability to manipulate the spin of protons. Based on this principle, it provides superior soft tissue contrast compared to other modalities, becoming suitable for assessing deep infiltration of the primary tumor and separation
A

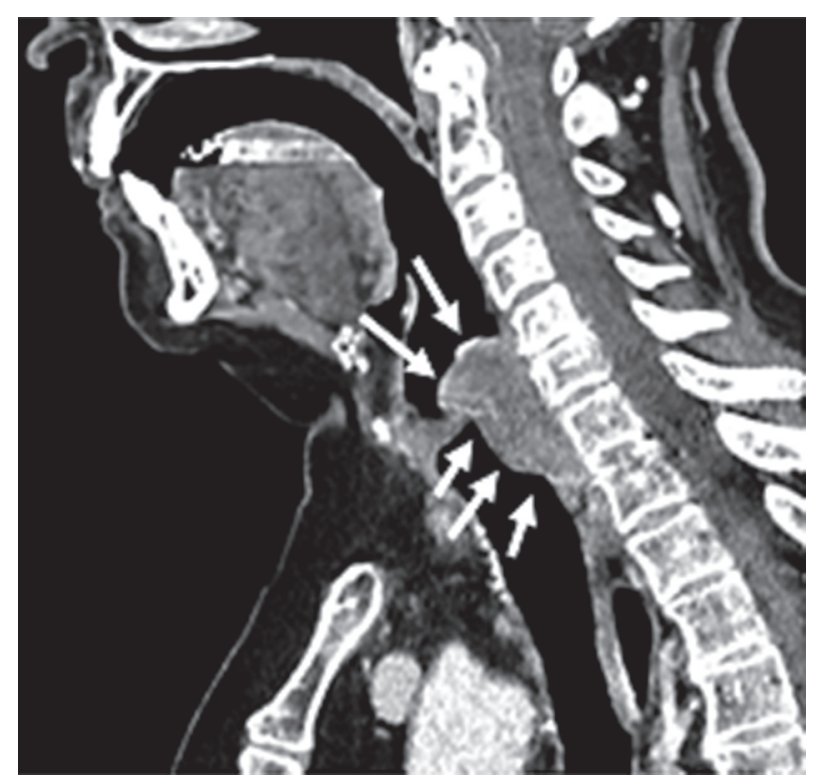

B

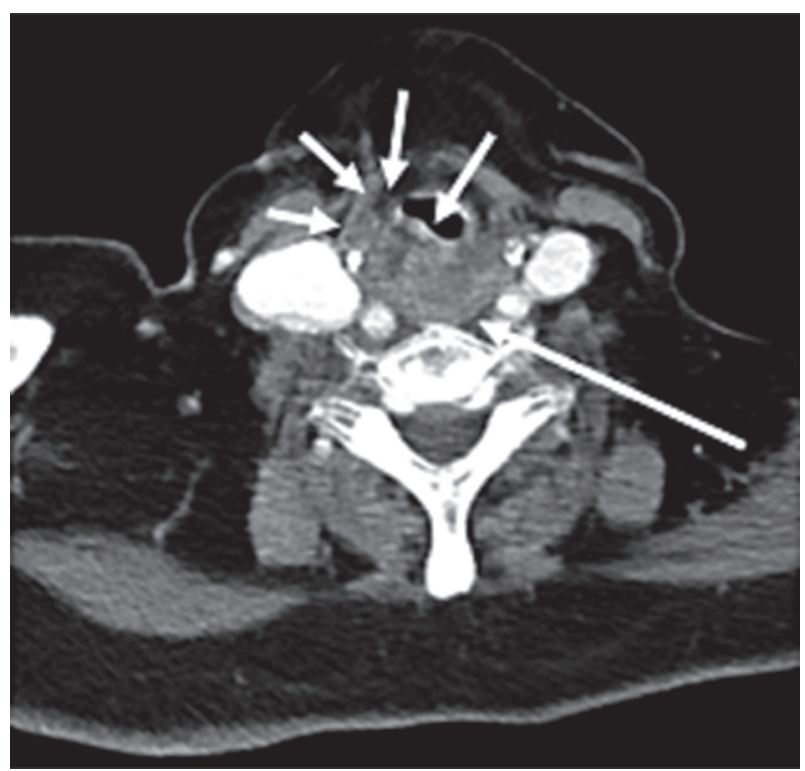

Fig. 1. Contrast-enhanced CT neck scan demonstrating malignancy of the hypopharynx. A) Sagittal view and B) axial view. A) Sagittal CT imaging shows enhancing infiltrating soft tissue thickening, along with the post-cricoid posterior hypopharyngeal wall, with extension above and below the vocal cords. B) Axial CT imaging shows that at the level of the false vocal cords, there is heterogeneously enhancing bulky soft tissue in the posterior hypopharyngeal wall, with extension and infiltration into the paraglottic fat anteriorly 
of oedema from tumor infiltration [10]. It can also provide assessment of bone marrow invasion and regional nodal disease. This is the preferred modality for local assessment of suprahyoid neck that include nasopharynx, sinuses, oral cavity, and oropharynx.

T1-weighted sequences provide excellent anatomical assessment due to increased conspicuity of fat planes, bone marrow signal, and visible lymph node capsule. Using the principle of delineating fat planes, it provides optimal evaluation of the nasopharynx, parapharyngeal fat plane, floor of mouth, oropharynx, pre-epiglottic space, and false vocal cords. High/bright T1 signal is caused by fat, subacute blood products, such as methemoglobin, slow-flow blood, melanin, protein rich fluid, paramagnetic substances (iron, ferritin, hemosiderin, melanin), and occasionally calcification. Low/dark T1 signal is caused by fluid, air, hyperacute hemorrhage, densely calcified/ osseous lesions, fibrous tissue, and vascular flow voids.

T2-weighted short-tau inversion recovery (STIR) sequences are particularly of value in characterizing soft tissue and further differentiating post-treatment fibrosis from residual tumor. High/bright signal on T2 sequences include fluid, inflammation, infarction, and subacute blood products. Low/dark T2 signal is caused by fibrous tissue, calcification, protein-rich fluid, paramagnetic substances, and vascular flow voids. Therefore, bright T2 signal can highlight pathological lymph nodes and on occasion, tumor or post-treatment oedema. Using the same principle, this can also demonstrate infection and inflammatory processes [11]. STIR sequences further assist in evaluation by selectively diminishing the hyperintensity of fat, preventing the obscuration of subtle lesions.

The main features of T1, T2, and contrast MRI sequences in head and neck imaging are presented in Table 1.
Gadolinium sequences are of value in detecting subtle or occult early disease, which may not be visible on other modalities due to the dynamic vascular nature of malignant processes. It is used with fat saturation in at least one plane to accurately define tumor margins, particularly with respect to skull base and perineural extension. A volumetric post-gadolinium sequence can be considered for multiplanar image reconstruction and radiotherapy planning. Limitations to this technique include normal enhancement of pharyngeal and aerodigestive mucosa, which may conceal smaller lesions.

Diffusion-weighted imaging (DWI) has an evolving role in head and neck cancer, especially in defining tumor treatment response. This works on the principle that hypercellular tumors and pathological lymph node show diffusion restriction and hence, low apparent diffusion coefficient (ADC) values, while oedema, inflammatory, and necrotic processes present low cellularity and high ADC values.

Dynamic contrast-enhanced MRI perfusion imaging can be of value in detecting and quantifying a microvascularity within tissue, providing characterization for head and neck cancers. This is used to generate blood flow, blood volume, and transit time parameters within areas of interest, and may help in assessing the histological grade of tumors. It is currently a growing area of research and has yet to be used in multisite clinical trials for HNC. Similarly, MRI spectroscopy can be applied to a prescribed region of tissue, providing a molecular breakdown of biochemical composition and evaluating the presence of specific metabolites. Therefore, it has the potential to differentiate between non-malignant tissue, malignancy, and post-radiation changes. However, as a modality of imaging, MRI comparatively incurs higher

Table 1. The role of different magnetic resonance imaging (MRI) sequences in head and neck cancer imaging

\begin{tabular}{|c|c|c|c|}
\hline $\begin{array}{l}\text { MRI } \\
\text { sequences }\end{array}$ & $\mathrm{T} 1$ & $\mathrm{~T} 2$ & Contrast \\
\hline Bright & $\begin{array}{l}\text { - Fat } \\
\text { - Subacute blood products (methemoglobin) } \\
\text { - Slow-flow blood } \\
\text { - Melanin } \\
\text { - Protein rich fluid } \\
\text { - Paramagnetic substances (iron, ferritin, } \\
\text { hemosiderin) } \\
\text { - Calcification }\end{array}$ & $\begin{array}{l}\text { - Fluid } \\
\text { - Inflammation } \\
\text { - Infarction } \\
\text { - Subacute blood products }\end{array}$ & $\begin{array}{l}\text { - Tumor } \\
\text { - Pathological lymph nodes } \\
\text { - Acute/subacute post-treat- } \\
\text { ment changes } \\
\text { - Inflammatory processes } \\
\text { - Infection }\end{array}$ \\
\hline Dark & $\begin{array}{l}\text { - Fluid } \\
\text { - Air } \\
\text { - Hyperacute hemorrhage } \\
\text { - Densely calcified/osseous lesions } \\
\text { - Fibrous tissue } \\
\text { - Vascular flow voids }\end{array}$ & $\begin{array}{l}\text { - Fibrous tissue } \\
\text { - Calcification } \\
\text { - Protein-rich fluid } \\
\text { - Paramagnetic substances } \\
\text { - Vascular flow voids }\end{array}$ & \\
\hline $\begin{array}{l}\text { Role } \\
\text { in imaging }\end{array}$ & $\begin{array}{l}\text { - Delineation of fat planes and optimal evalua- } \\
\text { tion of the nasopharynx, parapharyngeal fat } \\
\text { plane, floor of mouth, oropharynx, } \\
\text { pre-epiglottic space, and false vocal cords } \\
\text { - Assessment of bone marrow signal } \\
\text { - Assessment of visible lymph node capsule }\end{array}$ & $\begin{array}{l}\text { - Assessment of primary tumor } \\
\text { - Assessment of pathological } \\
\text { lymph nodes and extracapsular } \\
\text { disease spread } \\
\text { - Post-treatment changes } \\
\text { - Inflammatory processes }\end{array}$ & $\begin{array}{l}\text { - Delineation of primary tumor } \\
\text { - Assessment of perineural } \\
\text { spread } \\
\text { - Assessment of pathological } \\
\text { lymph nodes }\end{array}$ \\
\hline
\end{tabular}


costs and longer scan acquisition times due to the number of images and complexity of reconstruction [12]. Therefore, it is subjected to motion of artefacts resulting from breathing or swallowing. There are numerous limitations, including renal impairment, metal fragments/implants/ prostheses, older pacemakers, and ill patients.

With regards to MRI scan performance, there are multiple absolute contraindications, which each patient should be assessed for, by undergoing a screening questionnaire. These include cardiac implantable electronic devices (CIED), such as pacemakers, implantable cardioverter defibrillators (ICDs), and cardiac resynchronization therapy (CRT) devices, metallic intraocular foreign bodies and fragments, implantable neurostimulation systems, cochlear implants/ear implant, drug infusion pumps and metallic cerebral artery aneurysm clips [13]. Few departments allow imaging of pacemakers and ICDs at a minimum of 6 weeks after a placement in a 1.5 T MRI scanner under strict conditions. This should be assessed alongside local departmental policy in accordance with the brand and type of MRI scanner, and with individual discussion of each case with the relevant cardiology team [14].

There are a few absolute contraindications to gadolinium contrast medium injection, which include severe renal disease $\left(\mathrm{eGFR}<30 \mathrm{ml} / \mathrm{min} / 1.73 \mathrm{~m}^{2}\right)$, acute deteriorating renal function, pregnancy, and previous anaphylactic/ anaphylactoid reaction to gadolinium contrast agent [15].

In the presence of a contraindication to MRI contrast or low suspicion for malignancy, a limited non-contrast MRI can be performed with T1, T2, STIR, and diffusion- weighted sequences. This can provide an assessment of disruption to fat planes, nasopharynx, oral cavity, oropharynx, pre-epiglottic space, false vocal cords, lymph nodes, and bone marrow signal. Example of contrastenhanced MRI neck is presented in Figure 2.

\section{Positron emission tomography combined with computed tomography}

Positron emission tomography (PET) is combined with whole body CT for anatomical detail using the radiolabeled 18 fluorodeoxyglucose (FDG) tracer to demonstrate areas of abnormal, increased metabolic activity. It works by the principle that the positron emitting glucose analogue is taken up by abnormal highly metabolically active cancerous cells and therefore, FDG concentration and distribution can be directly measured and correlated with primary or metastatic disease. This would help to differentiate cancerous tissue from post-treatment oedema, scarring, and benign lesions. Experienced radiologists can interpret these scans through qualitative visual assessment.

The recent UK National Institute of Clinical Excellence and USA American Joint Committee on Cancer guidelines now recommend FDG PET-CT as a part of staging for head and neck squamous cell carcinoma (HNSCC), if there is an evidence of cancer spread beyond the primary site. These include patients with stage III and IV HNSCC $[16,17]$ and those diagnosed with nasopharyngeal cancer in the UK. In patients who have undergone chemoradiotherapy, it is the most sensitive modality for assessing
A

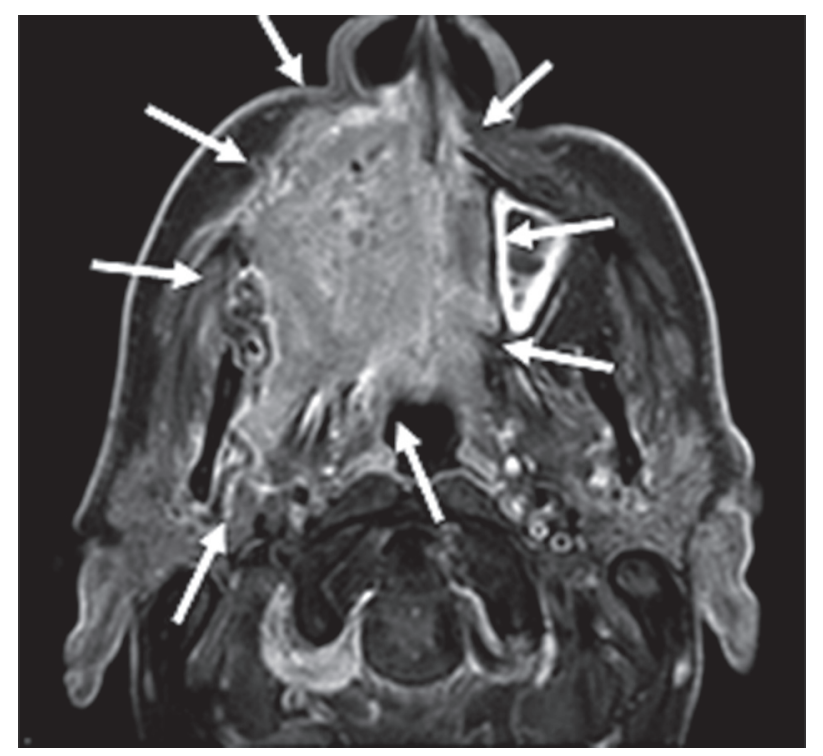

B

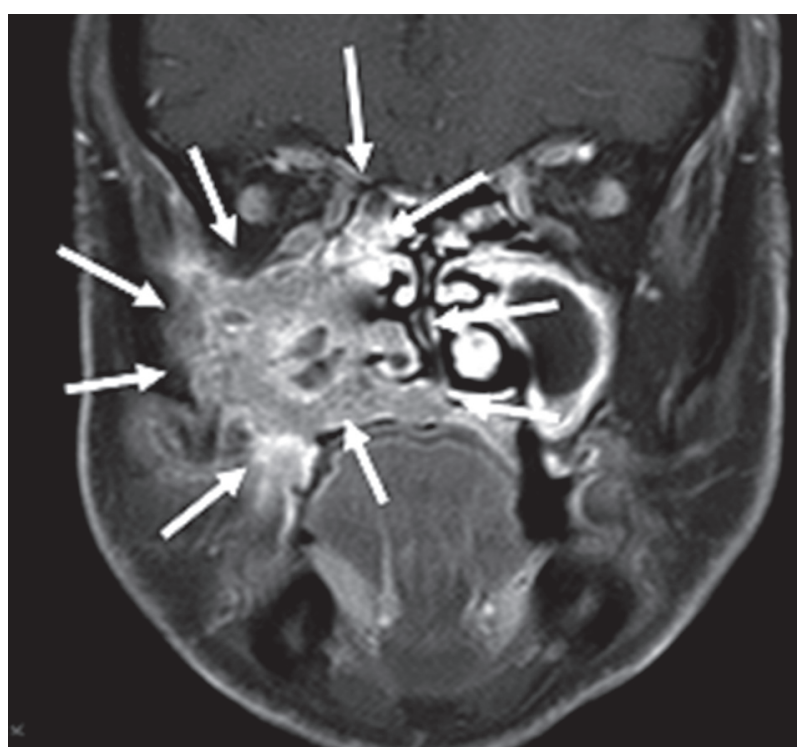

Fig. 2. Contrast-enhanced MRI neck scan demonstrating an extensively infiltrating necrotic malignancy centered in the right maxillary sinus, with heterogeneously bright contrast enhancement. A) Axial MRI imaging demonstrates a large avidly enhancing tumor centered in the right maxillary sinus. This extends anteriorly and laterally through the maxillary walls to the skin surface involving the right nasolabial fold and the facial muscles. Posteriorly, this extends into the pterygopalatine fossa, post-nasal space, and right masticator space infiltrating the right medial and latent pterygoid muscles. B) Coronal NIRI imaging demonstrates a large enhancing tumor centered in the right maxillary sinus, with non-enhancing areas of necrosis. This extends medially into the nasal cavity involving right middle and inferior nasal turbinate, along with infiltration of the right hard palate inferiorly 
post-treatment disease recurrence, when performed at 3 to 6 months following chemoradiotherapy. Additionally, it can be used to identify an unknown primary in the presence of neck lymphadenopathy and no obvious primary site on CT/MRI.

Disadvantages of PET include high cost and time consuming, with the amount of preparation required and investigation time. PET interpretation can also be nonspecific to malignancy, as inflammatory processes demonstrate increased metabolic activity.

There are a few contraindications to undergoing a PET-CT scan, such as pregnancy, uncontrolled diabetes, and patient inability to cooperate with the scan process that involves lying in supine position for 30-60 minutes and keeping still for 1-2 hours [18].

Recently, there has been a development of new PET radiotracers, which include amino acid radiotracers, specific for detection of HNSCC, radiolabeled antibodies for tumor angiogenesis characterization, and PET with 18 [F] fluorothymidine as a marker of tumor hypoxia. However, these require systematic clinical studies before they can be used for patient management. Example of FDG PET-CT in a case of cancer of unknown primary is presented in Figure 3.

\section{Ultrasound}

Ultrasound is easily accessible and can provide excellent non-invasive soft tissue characterization when assessing superficial primary sites and nodal basins. In head and neck imaging, these include neck lumps, salivary gland lesions, thyroid nodules, and regional lymph node morphology. Patients are often referred from general practice or after ear, nose, and throat (ENT)/endocrine specialized clinical review. The technique of ultrasound is operator dependent, and localized radiological grading of thyroid nodules is solely related to ultrasound morphology features in accordance with the American College of Radiology Thyroid Imaging Reporting and Data System (TI-RADSTM) guidelines.

Due to its ability to produce quick results and provide real-time guidance for fine needle aspiration cytology (FNAC), it can play an essential role in speeding up the diagnostic pathway as a part of one-stop clinic, established in some centers in the UK. This is where patients are evaluated in ENT clinic and if considered necessary, proceeded to have prompt ultrasound \pm FNAC and re-reviewed after rapid cytological assessment on the same day. Following the procedure of FNAC or core biopsy, it is recommended to monitor every patient on anticoagulation in the department for 30-45 minutes to prevent any possible complication, such as neck swelling or hematoma.

For salivary gland lesions, thyroid nodules, and lymph nodes, ultrasound provides excellent image resolution and it is the preferred modality of imaging for guided diagnostic interventional procedures, such as FNAC and core needle biopsy, due to the superficial nature of these sites. The limitations of ultrasound are its reliance on operator skills and inability to assess deeper structures due to limited soft tissue penetration and overlying bone or air artefact. CT-guided biopsy can rarely be considered, when there is an inadequate acoustic window with deep-seated head and neck lesions covered by artefact. Examples of ultrasound scan are presented in Figure 4.
A

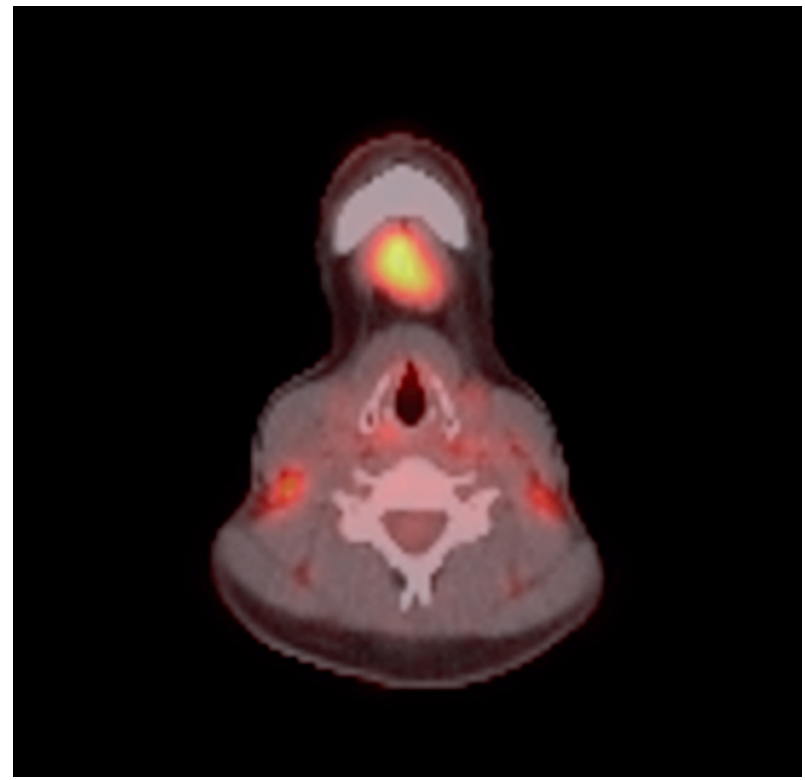

B

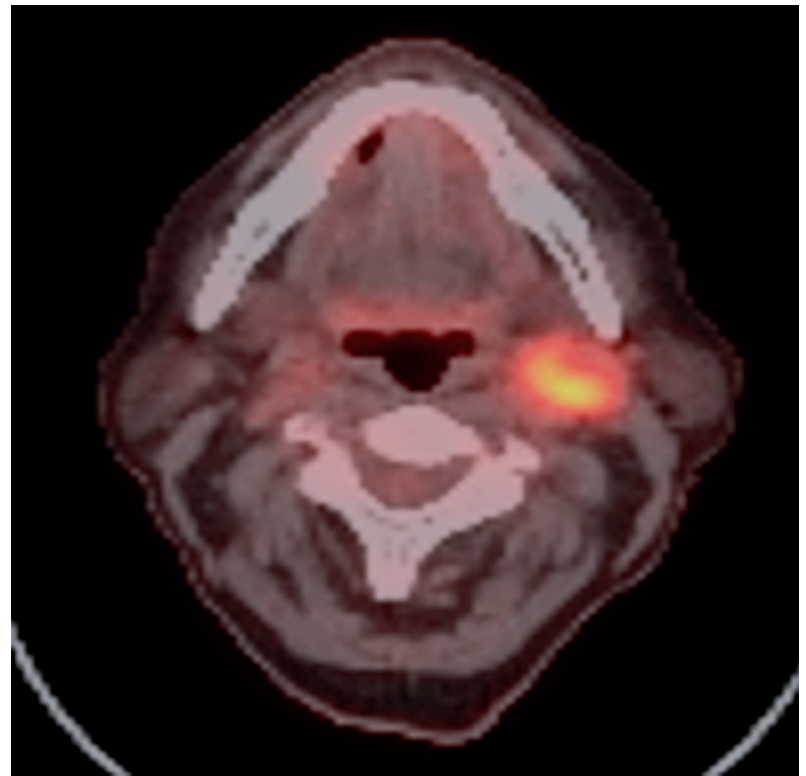

Fig. 3. FDG PET-CT scan selected axial slices, which demonstrate high grade FDG activity within a submental IA lymph node (A) and left level 2 lymph node (B) in a case of unknown primary cancer. A) Axial PET-CT imaging demonstrates high grade FDG activity within a submental 1A lymph node. B) Axial PET-CT imaging demonstrates high grade FDG activity within a left level 2 lymph node 
A

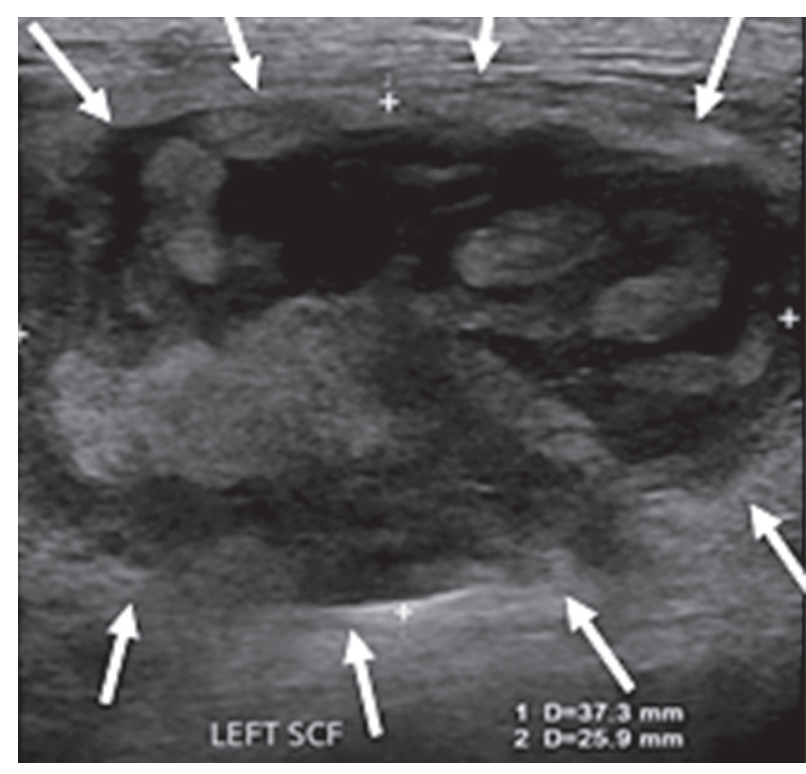

B

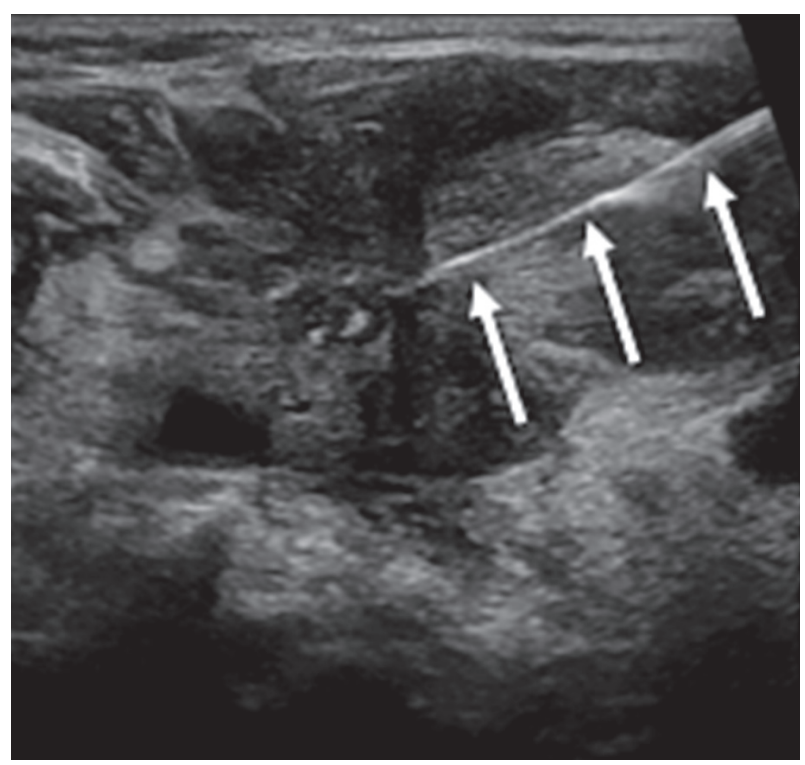

Fig. 4. A) Ultrasound demonstrating measurements of a large partially solid-cystic mass of lymph nodes in the left supraclavicular fossa (SCF). B) A fine needle aspiration was subsequently performed. Cytology results confirmed metastatic squamous cell carcinoma. A $25 \mathrm{G}$ needle is delineated entering the left SCF lesion, whilst performing a fine needle aspiration

Table 2. Comparison of different imaging modalities used in head and neck oncology

\begin{tabular}{|c|c|c|c|c|c|}
\hline $\begin{array}{l}\text { Imaging } \\
\text { modality }\end{array}$ & Principle & Duration & Contrast & $\begin{array}{c}\text { Minimum } \\
\text { staff required }\end{array}$ & Limitations \\
\hline Ultrasound & $\begin{array}{l}\text { High-frequency } \\
\text { sound waves }\end{array}$ & $\begin{array}{l}20-30 \\
\text { minutes }\end{array}$ & None & 3 & $\begin{array}{l}\text { - Reliance on operator skills } \\
\text { - Inability to assess deeper structures due to limited soft } \\
\text { tissue penetration and overlying bone or air artefact }\end{array}$ \\
\hline CT & $\begin{array}{l}\text { Series of cross- } \\
\text { sectional X-ray } \\
\text { images acquired } \\
\text { through sections } \\
\text { of the body }\end{array}$ & $\begin{array}{l}15-30 \\
\text { minutes }\end{array}$ & $\begin{array}{l}\text { Omnipaque } \\
300 \mathrm{Mg} \\
\text { lodine }^{\circledR}\end{array}$ & $2-3$ & $\begin{array}{l}\text { - Radiation dose burden } \\
\text { - Risk of contrast-induced nephropathy } \\
\text { - Artefacts secondary to dental amalgam/ orthopedic } \\
\text { prostheses } \\
\text { - Absolute contraindications to contrast, such as } \\
\left.\text { severe renal disease (eGFR < } 30 \mathrm{ml} / \mathrm{min} / 1.73 \mathrm{~m}^{2}\right) \text {, } \\
\text { acute liver disease, asthma, thyroid disease, dehy- } \\
\text { dration, sickle cell anemia, seizures, phaeochromocy- } \\
\text { toma, and allergies to contrast }\end{array}$ \\
\hline MRI & $\begin{array}{l}\text { Electromagnetic } \\
\text { field and radio } \\
\text { waves }\end{array}$ & $\begin{array}{c}\text { 30-45 } \\
\text { minutes }\end{array}$ & Gadolinium & $2-3$ & $\begin{array}{l}\text { - Claustrophobia } \\
\text { - Severe renal disease (eGFR < } 30 \mathrm{ml} / \mathrm{min} / 1.73 \mathrm{~m}^{2} \text { ) } \\
\text { - Pregnancy } \\
\text { - Gadolinium contrast allergy } \\
\text { - Cardiac implantable electronic devices (CIED), such } \\
\text { as pacemakers, implantable cardioverter defibrilla- } \\
\text { tors (ICDs) and cardiac resynchronization therapy } \\
\text { (CRT) devices (see text for detail) } \\
\text { - Metallic intraocular foreign bodies and fragments } \\
\text { - Implantable neurostimulation systems } \\
\text { - Cochlear implants } \\
\text { - Drug infusion pumps } \\
\text { - Metallic cerebral artery aneurysm clips }\end{array}$ \\
\hline FDG PET-CT & $\begin{array}{l}\text { Radiolabeled } 18 \\
\text { fluorodeoxyglu- } \\
\text { cose (FDG) tracer } \\
\text { combined with } \\
\text { whole body CT }\end{array}$ & $\begin{array}{l}\text { 90-120 } \\
\text { minutes }\end{array}$ & $\begin{array}{l}\text { Radiolabeled } \\
18 \text { fluorode- } \\
\text { oxyglucose } \\
\text { (FDG) tracer }\end{array}$ & $3-4$ & $\begin{array}{l}\text { - Pregnancy } \\
\text { - Avoidance of breast feeding for } 24 \text { hours } \\
\text { - Persistent radioactivity for } 12 \text { hours } \\
\text { - Contra-indication in uncontrolled diabetes } \\
\text { - Limitations in patients with inability to lie still } \\
\text { for } 1-2 \text { hours }\end{array}$ \\
\hline
\end{tabular}




\section{Summary}

As presented in this article, there are multiple modalities of imaging, with corresponding functions in diagnosis, staging, and management of head and neck cancers (Table 2).

Contrast-enhanced CT is readily available and is considered the gold standard for routine tumor-nodemetastasis staging as well as assessing bone detail and involvement.

MRI has the superior ability to characterize localized disease extent and soft tissue involvement for most of the primary subsites as described above.

Ultrasound is commonly used to assess thyroid nodules, salivary gland lesions, and superficial lymph nodes.

Diagnostic fine needle aspiration and core biopsy procedures of lesions and lymph nodes are performed under real-time ultrasound guidance. PET-CT has a great role in assessing known advanced and unknown primary disease and is often the first post-treatment baseline imaging at 12 weeks to assess therapy response.

All these modalities of imaging have a strong influence on treatment planning and therefore, an understanding of the merits of each type and timing of optimal imaging for anatomical characterization and accurate staging is essential.

\section{Disclosure}

The authors report no conflict of interest.

\section{References}

1. Sankaranarayanan R, Masuyer E, Swaminathan R et al. Head and neck cancer: a global perspective on epidemiology and prognosis. Anticancer Res 1998; 18: 4779-4786.

2. Kovács G, Martinez-Monge R, Budrukkar A et al. GEC-ESTRO ACROP recommendations for head \& neck brachytherapy in squamous cell carcinomas: 1st update - Improvement by cross sectional imaging based treatment planning and stepping source technology. Radiother Oncol 2017; 122: 248-254.

3. $\mathrm{Hu} \mathrm{H}, \mathrm{He} \mathrm{HD}$, Foley WD, Fox SH. Four multidetector-row helical CT: image quality and volume coverage speed. Radiology 2000; 215: 55-62.

4. Cantrell SC, Peck BW, Li G et al. Differences in imaging characteristics of HPV-positive and HPV-negative oropharyngeal cancers: a blinded matched-pair analysis. Am J Neuroradiol 2013; 34: 2005-2009.

5. Royal College of Radiologists (UK). Recommendations for cross-sectional imaging in cancer management, Second edition. Head and neck cancers. June 2014. https://www.rcr. ac.uk/system/files/publication/field_publication_files / BFCR\%2814\%292_Head\%26neck.pdf (accessed 4 Feb 2020).

6. Smith-Bindman R, Lipson J, Marcus R et al. Radiation dose associated with common computed tomography examinations and the associated lifetime attributable risk of cancer. Arch Internal Med 2009; 169: 2078-2086.

7. GOV.UK. Patient dose information: guidance. Published 4 September 2008. https:/ / www.gov.uk/government/publications/medical-radiation-patient-doses/patient-dose-information-guidance (accessed 5 Feb 2020).

8. United States Environmental Protection Agency (EPA). Radiation sources and doses. https://www.epa.gov/radiation/radiation-sources-and-doses\#backgroundradiation (accessed 5 Feb 2020).
9. WebMD. Who should not take Omnipaque $300 \mathrm{Mg}$ Iodine/Ml Intravenous Solution? https://www.webmd.com/drugs/2/ drug-151518/omnipaque-300-intravenous/details/list-contraindications (accessed 5 Feb 2020).

10. Taheri A, Vishwanath V, Wong J et al. The use of imaging and interventional radiology in modern oncology. Medicine 2020; 48: 73-78.

11. Johnson KA. Basic proton MR imaging. http://www.med. harvard.edu/AANLIB/basicsMR.html (accessed 5 Feb 2020).

12. Tshering Vogel DW, Thoeny H. Cross-sectional imaging in cancers of the head and neck: how we review and report. Cancer Imaging 2016; 16: 20.

13. Ghadimi M, Sapra A. Magnetic Resonance Imaging (MRI), Contraindications. In: StatPearls. StatPearls Publishing, Treasure Island, FL 2020. https:/ / www.ncbi.nlm.nih.gov/books / NBK551669/ (accessed 5 Feb 2020).

14. University of California, San Francisco (UCSF). Pacemakers, ICDs, Pacing Wires and Loop Recorders. https://radiology.ucsf.edu/patient-care/patient-safety/mri/pacemakersicds\#accordion-specific-guidelines-for-patients-with-pacemakersicds (accessed 6 Feb 2020).

15. Ferris N, Goergen S. Gadolinium contrast medium (MRI contrast agents). https://www.insideradiology.com.au/gadolinium-contrast-medium-hp/ (accessed 5 Feb 2020).

16. National Institute for Health and Care Excelllence (NICE). Head and neck cancer. Quality standards [QS146]. Published 3 Mar 2017. https://www.nice.org.uk/guidance/qs146/chapter/Quality-statement-2-Clinical-staging (accessed 22 Sep 2019).

17. Plaxton NA, Brandon DC, Corey AS et al. Characteristics and limitations of FDG PET/CT for imaging of squamous cell carcinoma of the head and neck: a comprehensive review of anatomy, metastatic pathways, and image findings. AJR Am J Roentgenol 2015; 205: 519-531.

18. Alliance Medical. Specific clinical contraindications to PET-CT. https://www.alliancemedical.co.uk/sites/default/files/upload/images/Clinical\%20Contraindications \%20to\%20PET-CT. pdf (accessed 5 Feb 2020). 